\title{
A Magnetorheological Fluid Damper for Rotor Applications
}

\author{
P. Forte, M. Paternò, and E. Rustighi \\ Dipartimento di Ingegneria meccanica, Nucleare e della Produzione, University of Pisa, Pisa, Italy
}

Even though we are still far from industrial applications, in the last decade there has been increasing attention directed toward the employment of electrorheological (ER) and magnetorheological (MR) fluids in active bearings and active squeeze film dampers in rotordynamics. MR fluids react to magnetic fields undergoing reversible changes in their mechanical characteristics, viscosity, and stiffness in particular. In previous literature, some applications of ER fluids in rotor squeeze film dampers can be found; however, on the contrary, little is reported on similar test rigs set up for MR dampers. In this work, the design of an MR squeeze film damper is presented and discussed. A numerical simulation has been carried out in order to evaluate the dynamic behavior of the damped rotor as a function of the magnetic field strength. The test rig is made of a slender shaft supported by two oilite bearings and an unbalanced disk. The damper is interfaced with the shaft through a rolling bearing. Electric coils generate the magnetic field whose field lines cross the MR film. Since the damping characteristics can be varied continuously by controlling the magnetic field, it is possible to have the optimum conditions for each regime of rotational speed. Preliminary tests are encouraging.

Keywords Active damper, Critical speed, Magnetorheological fluid, Rotordynamics, Test rig

The problem of vibrations in rotordynamics is commonly faced with passive squeeze fluid film or elastomeric dampers. Unfortunately, however, the damping effect varies with the rotor speed; experimental evidence shows that in order to dissipate energy at the critical speeds, the rotor displacement in the damper has to be significant, meaning that viscosity must be

Received 22 January 2003; accepted 2 July 2003.

Address correspondence to P. Forte, DIMNP University of Pisa, Via Dioti Salvi 2, Pisa 56126, Italy. E-mail: p.forte@ing.unipi.it low. On the contrary, in non critical conditions, higher values are required (Bonneau and Frene, 1997). In the last decade there has been increasing attention directed toward the employment of electrorheological (ER) and magnetorheological (MR) fluids, suspensions of micron-sized dielectric/ferromagnetic particles respectively, which, when subjected to electric/magnetic fields, link in chain structures oriented parallel to the field direction. Therefore, these materials behave as solids as long as the shear stress is lower than a threshold value which depends on the field strength, as quasi-Newtonian fluids if the shear stress is higher. The change induced in the yield shear stress produces a variation in their apparent viscosity and stiffness. These 'smart' materials have been successfully used in valves, dampers, and clutches and adaptive structures (Morishita and Tamaki, 1993; Weiss et al., 1993; Shakeri et al., 1996; Carlson and Weiss, 1994) but we are still far from industrial applications in rotordynamics.

In previous literature, theoretical and experimental studies on the employment of ER fluids in active journal bearings and squeeze dampers are reported (Tichy, 1991; Tichy, 1993; Dimarogonas and Kollias, 1992; Jung and Choi, 1995; Morishita and An, 1996; Nikolakopoulos and Papadopoulos, 1998). More limited information is available regarding MR fluids. In fact, while general aspects and applications of MR fluids have been dealt with to some extent (Kordonsky, 1993; Jolly and Carlson, 1996; Jolly et al., 1998; Bolter and Janocha, 1997) showing their promising characteristics, it was only recently that some papers have appeared on rotor dampers (Zhu et al., 2001; Carmignani and Forte, 2001).

In this work, the theoretical modeling, the design, and the preliminary testing of a MR squeeze film damper are presented and discussed.

\section{MODELING OF THE MAGNETORHEOLOGICAL FLUID}

In this article, we follow the analysis performed by Tichy (1993) for the lubrication of the one-dimensional bearing illustrated in Figure 1, where $u \gg v, \partial / \partial x \gg \partial / \partial y$, and $w=\partial / \partial z=0$. 


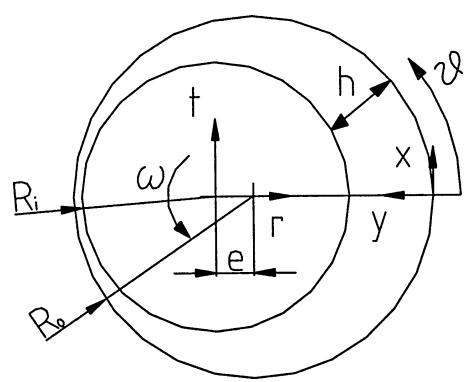

FIGURE 1

Schematic of damper geometry.

In this case, one obtains for the so-called Bingham model:

$\frac{\partial u}{\partial y}=0 \quad$ for $|\tau| \leq\left|\tau_{0}\right| ; \quad \tau=\tau_{x y}=\mu \frac{\partial u}{\partial y} \pm \tau_{0} \quad$ for $|\tau|>\tau_{0}$

meaning that for shear stress magnitude $|\tau|$ greater than a yield shear parameter $\tau_{0}$, the material flows as a Newtonian fluid with viscosity $\mu$, otherwise the material remains rigid. For the squeeze film damper, the film thickness is defined as:

$$
h=c(1+\varepsilon \cos \theta)
$$

where $c$ is the bearing radial clearance, $c=R_{0}-R_{i} \ll R_{0} \approx R$, $\varepsilon$ is the eccentricity ratio, $\varepsilon=e / c, \theta$ is the angular coordinate measured from the maximum film thickness location, and $x=$ $R \theta$. Therefore, the selected coordinate system rotates with the journal at a constant speed $\omega$. For any non-zero $\tau_{0}$, the implicit form of the Reynolds equation is obtained:

$\left(h \frac{d p}{d x}\right)^{3}+3\left(\frac{d p}{d x}\right)^{2}\left[4 \mu(\omega R h+q) \pm h^{2} \tau_{0}\right]-\left( \pm 4 \tau_{0}\right)^{3}=0$

The $+\tau_{0}$ condition is used when $d p / d x$ is negative and vice versa. Equation (3), being a cubic equation, has a standard analytical solution for $d p / d x$. However, any of the three roots may be the physically meaningful one, depending on the value of the parameters. The pressure is determined by integrating the pressure gradient expression and adjusting the constant flow rate $q$ so as to satisfy the periodicity condition $p(0, q)=$ $p(2 \pi, q)$ :

$$
p(\theta, q)=\int_{0}^{\theta} \frac{d p}{d \theta} d \theta+p(0, q) .
$$

Figure 2 shows typical dimensionless velocity profiles $\left(u^{*}=\right.$ $\left.u / U, y^{*}=y / h\right)$ for different values of the dimensionless yield shear stress parameter $\tau_{0}^{*}=\tau_{0} c /(\mu U)$. Both surfaces are considered as sliding in the reverse direction with $U_{1}=U_{2}=-U$. The flow is symmetric about the midplane $y=h / 2$, where the shear stress is zero. In the Newtonian case, a Poiseuille flow takes place with a uniform negative velocity superimposed. As the yield stress $\tau_{0}$ increases, a floating core is observed thickening

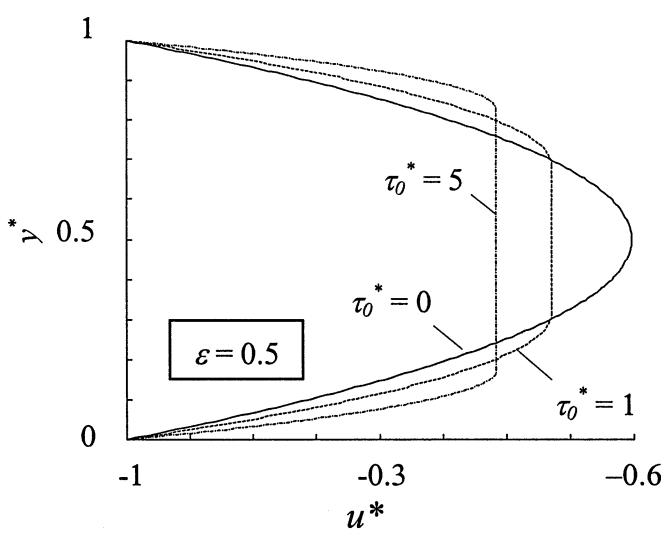

FIGURE 2

Typical velocity profiles.

about the midplane and filling the gap at two locations where $h / c=q^{*}$.

The radial and tangential forces on the rotor due to the film pressure (see Figure 1) are found by performing the following integrations:

$$
F_{r}=R L \int_{\pi}^{2 \pi} p \cdot \cos \theta d \theta \quad F_{t}=R L \int_{\pi}^{2 \pi} p \cdot \sin \theta d \theta
$$

in which simple cavitation conditions are assumed ( $\pi$ film). In order to obtain some data to design the actual damper, the dimensional form is preferable and the values reported in Table 1 were assumed. Since the damper clearance has to be rather large for various reasons (see the section "Description of the Device"), the assumption of small clearance of the theoretical analysis is not satisfied. Therefore the results of the numerical simulation are to be considered qualitative indications but not quantitatively comparable to experimental results.

The fluid used is the oil-based MR fluid MRF 132-LD produced by the Lord Corporation. An average value for the viscosity was considered neglecting the so-called 'shear thinning effect', i.e., the relationship between viscosity and shear rate.

Radial and tangential forces were calculated considering different values for the yield stress $\left(\tau_{0}=500,10000,20000 P a\right)$ and for the whirling velocity $(\omega=50,500,1000 \mathrm{rad} / \mathrm{sec})$.

Both the radial and tangential forces increase with the yield shear stress. Thus the tangential force has a finite value when $\varepsilon \rightarrow 0$. Such behavior can be explained considering Bingham's fluid constitutive equation. Moreover, both components increase with the whirling velocity.

TABLE 1

Numerical Values Used in Damper Calculation

\begin{tabular}{cccc}
\hline$L$ & $c$ & $R$ & $\mu$ \\
\hline $2 \mathrm{~cm}$ & $2.5 \mathrm{~mm}$ & $3.2 \mathrm{~cm}$ & $0.18 \mathrm{~Pa} \cdot \mathrm{s}$ \\
\hline
\end{tabular}


Tangential Force:

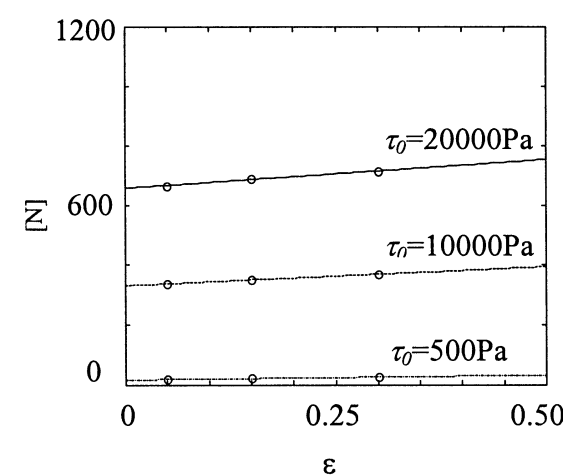

$\omega=50 \mathrm{rad} / \mathrm{sec}$

\section{$\underline{\text { Radial Force }}$}

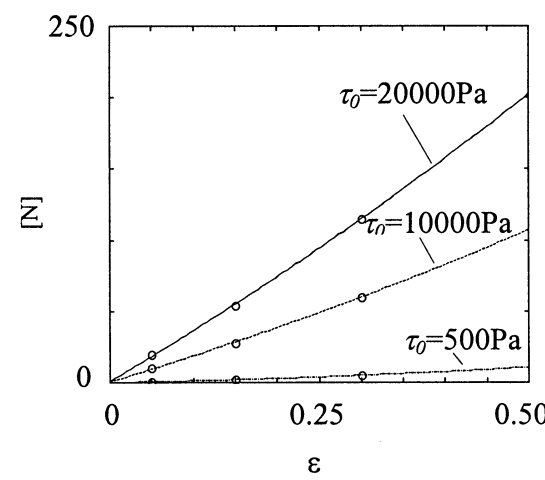

$\omega=50 \mathrm{rad} / \mathrm{sec}$

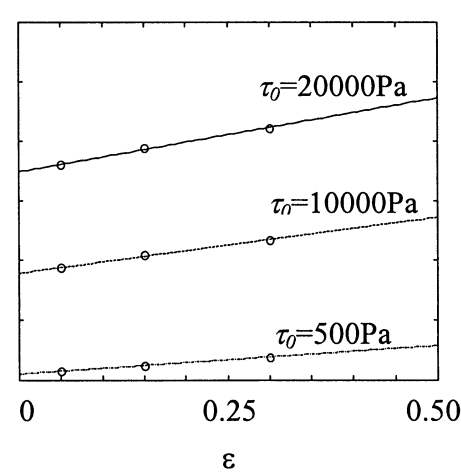

$\omega=500 \mathrm{rad} / \mathrm{sec}$

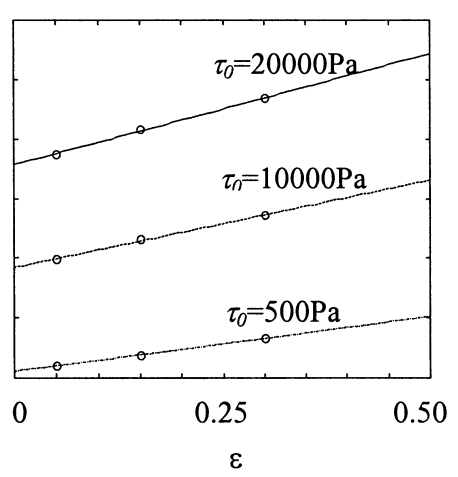

$\omega=1000 \mathrm{rad} / \mathrm{sec}$

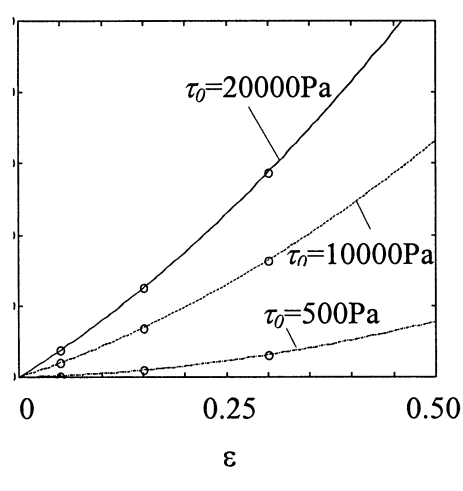

$\omega=500 \mathrm{rad} / \mathrm{sec}$

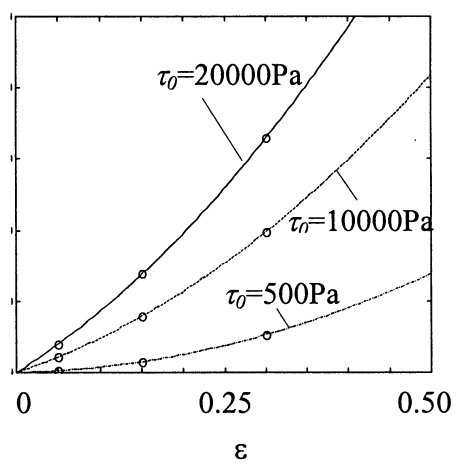

$\omega=1000 \mathrm{rad} / \mathrm{sec}$

\section{FIGURE 3}

Radial and tangential forces due to the pressure field in the gap. and $F_{r}$ :

Figure 3 suggests the following analytical expressions for $F_{t}$

$$
\begin{aligned}
& F_{t}=\left[a_{t}\left(\omega, \tau_{0}\right)+b_{t}\left(\omega, \tau_{0}\right) \cdot\left(\frac{e}{c}\right)\right] \\
& F_{r}=\left[a_{r}\left(\omega, \tau_{0}\right) \cdot\left(\frac{e}{c}\right)^{2}\right]
\end{aligned}
$$

\section{MODELING OF THE ROTOR-DAMPER SYSTEM}

In Figure 4, the schematic model of the rotor-damper system is presented. It is made of an elastic shaft, hinged at both ends, with a disk of mass $m_{d}$ connected in the middle.

The shaft is also supported by the squeeze-film damper. Two coordinate systems rotating with the shaft at its whirling velocity $\omega$ are defined. The center of mass of the disk is off- set by a distance $\delta$ from the geometric center of the shaft due to the unbalance, $m_{g}$. Thus, the unbalance vector makes a constant arbitrary angle $\beta$ with respect to the $X_{1}$ direction. The coordinates $\left(x_{1}, y_{1}\right)$ describe the displacement of the geometric center of the disk while $x_{2}$ describes the radial displacement of the inner cylinder of the damper (see the next section for the description of the device). The damper is accounted for by adding a second mass, $M_{2}$, to the shaft.

The tangential force due to the pressure generated in the damper acts in the $Y_{2}$ direction while the radial force acts in the $X_{2}$ direction. The inner cylinder of the damper is constrained by a "squirrel cage" (see the next section), which is accounted for in the analytical model by adding a radial stiffness, $k_{s c}$. The four unknown values $x_{1}, y_{1}, x_{2}$, and $\beta$ can be found by writing and solving the four equilibrium equations written in the $X_{1}, X_{2}, Y_{1}$, and $Y_{2}$ directions. In these equations we consider the centrifugal forces due to the masses $M_{1}$ and $M_{2}$, the elastic reactions of the shaft (using the linearized stiffness coefficient $k_{11}, k_{12}, k_{22}$ ), and 


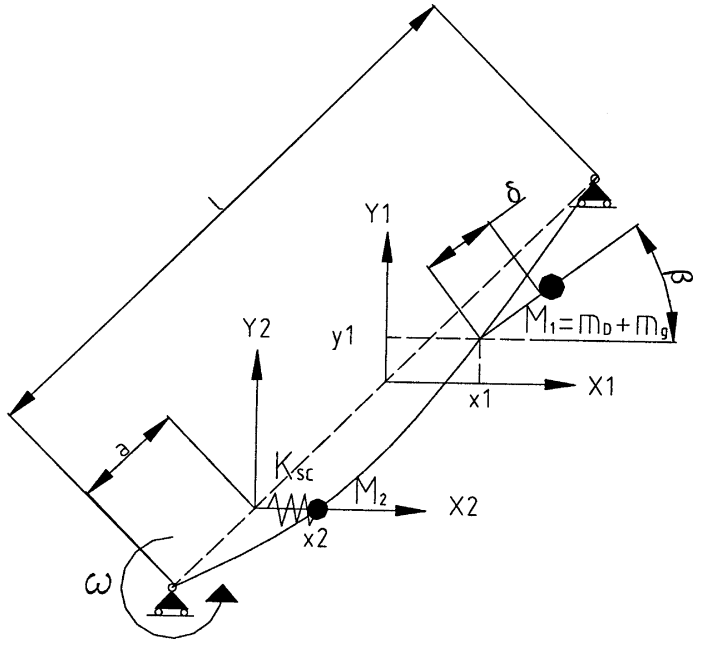

FIGURE 4

Schematic model of the rotor-damper system.

the possible damper reaction:

$$
\left\{\begin{array}{l}
k_{11} x_{1}+k_{12} x_{2}=M_{1} \omega^{2}\left(x_{1}+\delta \cos \beta\right) \\
k_{11} y_{1}=M_{1} \omega^{2}\left(y_{1}+\delta \sin \beta\right) \\
k_{12} x_{1}+k_{22} x_{2}+k_{s c} x_{2}+F_{r}\left(\omega, \tau_{0}\right)=M_{2} \omega^{2} x_{2} \\
k_{12} y_{1}+F_{t}\left(\omega, \tau_{0}\right)=0
\end{array}\right.
$$

Equations (7) are solved for several values of the yield stress and angular velocities varying from 0 up to $10000 \mathrm{rpm}$. That is actually the range of velocities which can be explored using the Bently Nevada Rotor Kit (see section "the test rig").

For every couple of values of $\omega$ and $\tau_{0}$, four solutions are found among which the physically meaningful one is chosen. When no meaningful solution arises it means that the forces due to unbalance are not high enough to break the columnar structures created in the MR fluid and the damper acts on the shaft as a rigid constraint. The numerical values used in the simulation are reported in Table 2.

In Figure 5, the radius of the shaft orbit, at section 1, versus the shaft angular velocity is shown. The results shown suggest two different ways to control the shaft dynamic instability:

1. A simple on-off procedure consisting in turning the device on while the shaft is passing through the critical speed and turning it off when the displacements start to increase again. When the device is active, the magnetic field applied on the fluid has to be high enough to maintain the fluid solid in every zone of the gap.
2. Setting the magnetic field at a constant value to obtain the shaft behavior shown in Figure 5 (yield stress $=5000 \mathrm{~Pa}$ ).

The limit of this calculation is that it does not consider the effects of the magnetic field on the mobile parts of the damper but the solution of this complex problem is beyond the scope of this work. The MR damper actually takes advantage of the combined effects of a variable viscosity squeeze film damper and of a magnetic damper. As regards the latter effect, the proposed device needs lower electric currents than with air gaps because the MR fluid has a magnetic permeability which is about five times that of air.

\section{DESIGN OF THE MR DAMPER}

\section{Description of the Device}

Figure 6 presents the longitudinal section of the device. It is entirely made of AISI 1040 steel except for the squirrel cage and the shaft bush made of an aluminium alloy. Each coil has 240 windings of a $\phi=0.63 \mathrm{~mm}$ wire capable of bearing a constant current of $1 A$, value that can rise up to $2 A$ for a limited time. The wires coming out of the coils are connected to each other so that the two coils are run by the current in opposite directions.

The rotation of the transversal section of the deformed shaft $(\phi=10 \mathrm{~mm})$ is allowed by a self-aligning double row ball bearing. The basic parameters of the MR film are the same shown in Table 1.

The radial clearance was set at a dimension of $c=2.5 \mathrm{~mm}$ after noticing that a smaller gap would have produced a damping effect with too low values of the yield stress, not taking advantage of the potential characteristics of the MR fluid. Moreover, due to the small dimensions of the Bently Nevada Rotor Kit used to test our device, it is impossible to create centrifugal forces high enough to be balanced by the pressure generated in a gap smaller than $2.5 \mathrm{~mm}$. Finally, with too small a gap, there is a higher risk of lock up of the damper due to the magnetic pull force between its moving parts.

Since one of the aims of this work was to verify the theoretical model, the damper was devised with one single gap and as long as possible in order to satisfy the one-dimensional bearing hypothesis and neglect the flow in the axial direction. Moreover, for the same reason, the gap was sealed by two flexible annular rubber membranes especially designed to have a negligible effect on the radial stiffness of the device. Anyway using a modular conception another version, involving two gaps and one coil has been set up, using almost the same components (the entire set of components is shown in Figure 7). That can be achieved by splitting the damper segment containing the gap into two

TABLE 2

Numerical Values for the Simulation of the Damped Operation

\begin{tabular}{|c|c|c|c|c|c|c|c|c|c|c|}
\hline$l$ & $a$ & $k_{11}$ & $k_{12}$ & $k_{22}$ & $k_{s c}$ & $m_{d}$ & $m_{g}$ & $M_{1}$ & $\delta$ & $M_{2}$ \\
\hline $2 \mathrm{~cm}$ & $11 \mathrm{~cm}$ & $452140 \mathrm{~N} / \mathrm{m}$ & $-539820 \mathrm{~N} / \mathrm{m}$ & $756200 \mathrm{~N} / \mathrm{m}$ & $174000 \mathrm{~N} / \mathrm{m}$ & $0.800 \mathrm{~kg}$ & $7 \mathrm{~g}$ & $0.807 \mathrm{~kg}$ & $2.625 \cdot 10^{-4} \mathrm{~m}$ & $0.400 \mathrm{~kg}$ \\
\hline
\end{tabular}




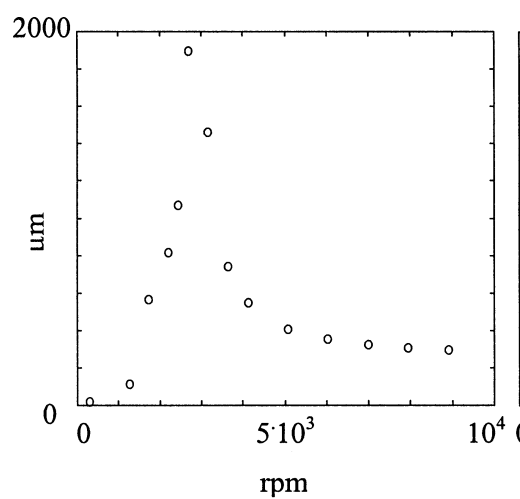

Free Shaft

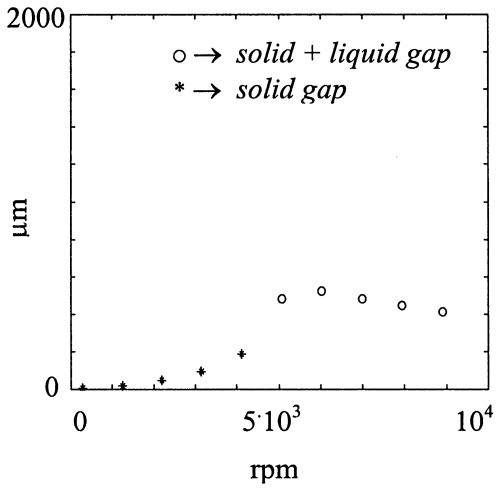

Yield Stress $=5000 \mathrm{~Pa}$

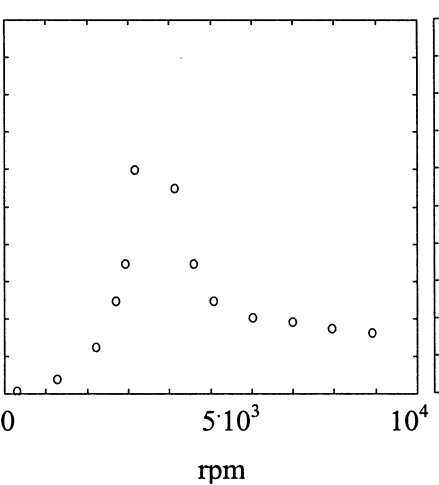

Newtonian Behavior

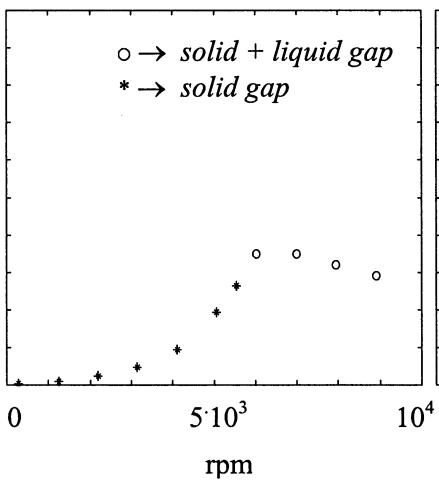

Yield Stress $=10000 \mathrm{~Pa}$

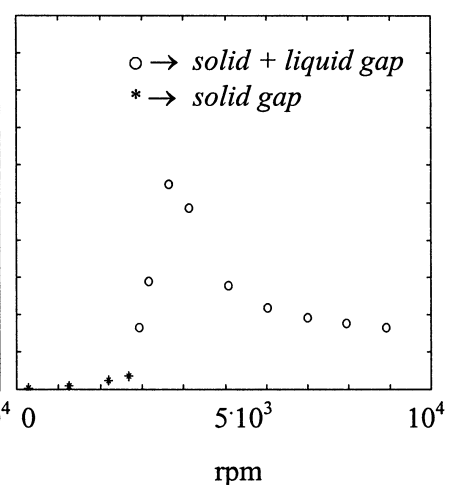

Yield Stress $=2500 \mathrm{~Pa}$

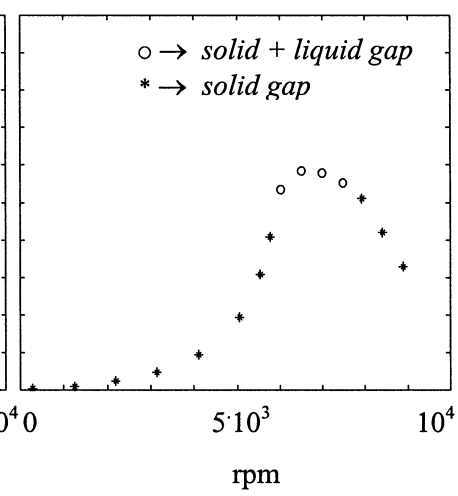

Yield Stress $=20000 \mathrm{~Pa}$

FIGURE 5

Effects of the fluid yield stress increase on the rotor behavior.

shorter segments and inserting a segment coil between the two. This latter configuration is similar to that recently tested by other authors (Zhu et al., 2001). The comparative analysis of the two configurations could be the object of future investigation.

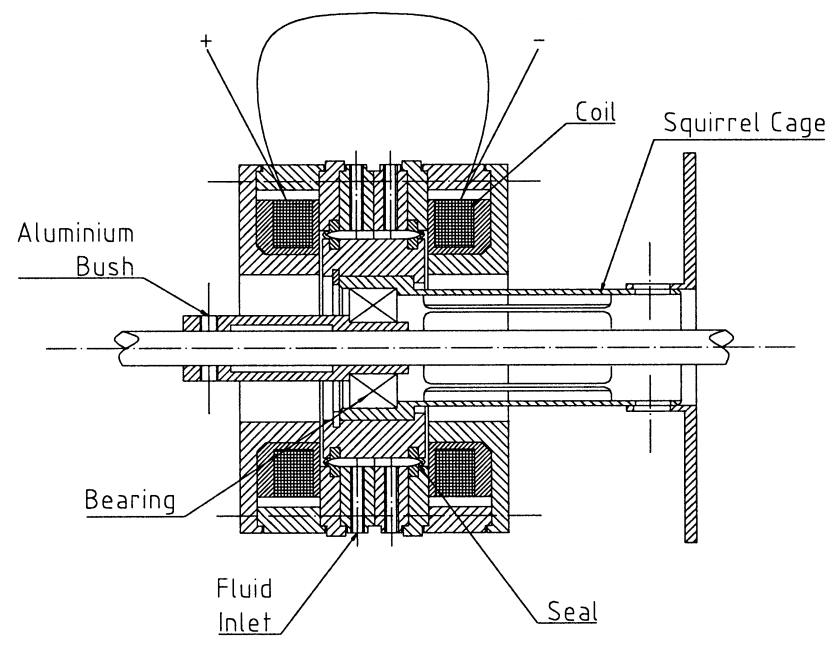

FIGURE 6

The MR fluid damper.

\section{Design of the Magnetic Circuit}

From the electromagnetic point of view, the damper structure can be considered as a circuit concentrating the magnetic field generated by the coils and guiding it through the MR fluid. An

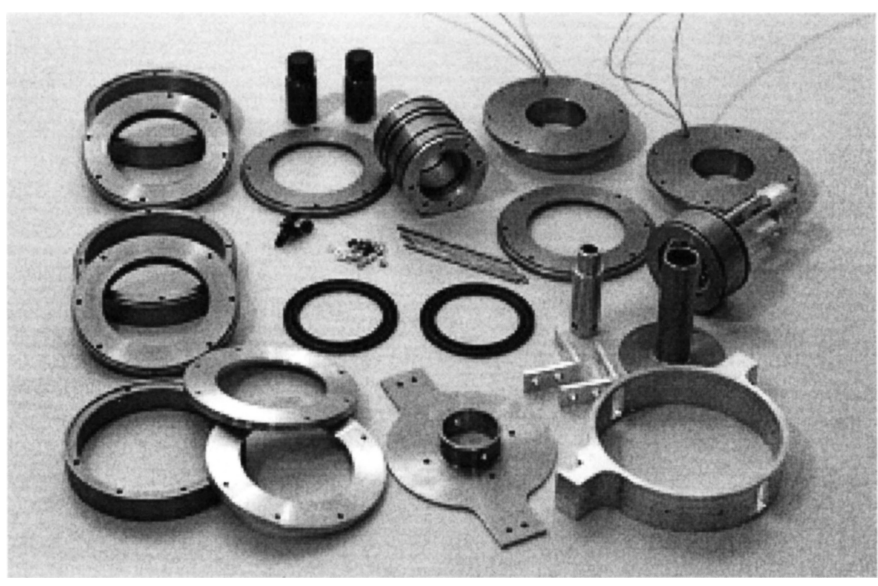

FIGURE 7

Components realized. 


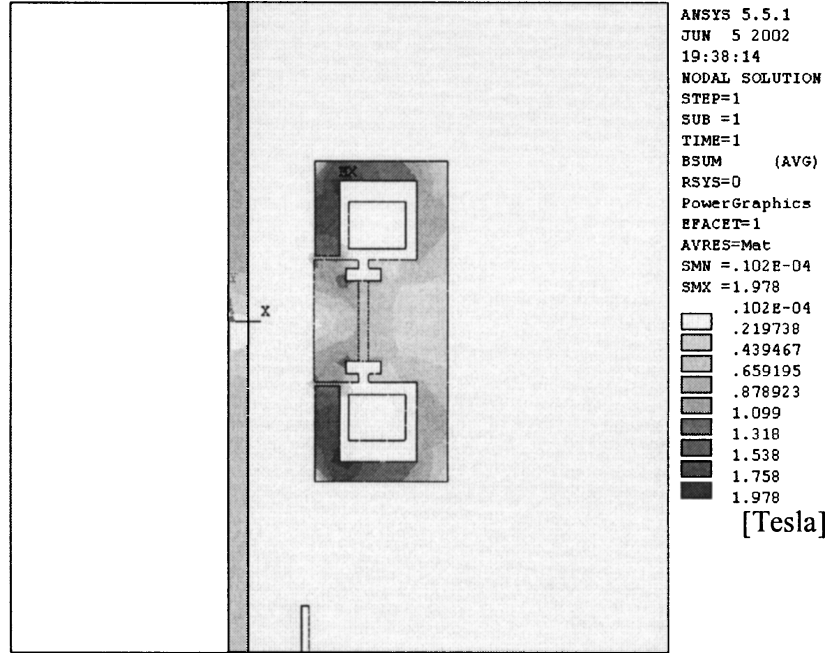

FIGURE 8

The magnetic field in the device.

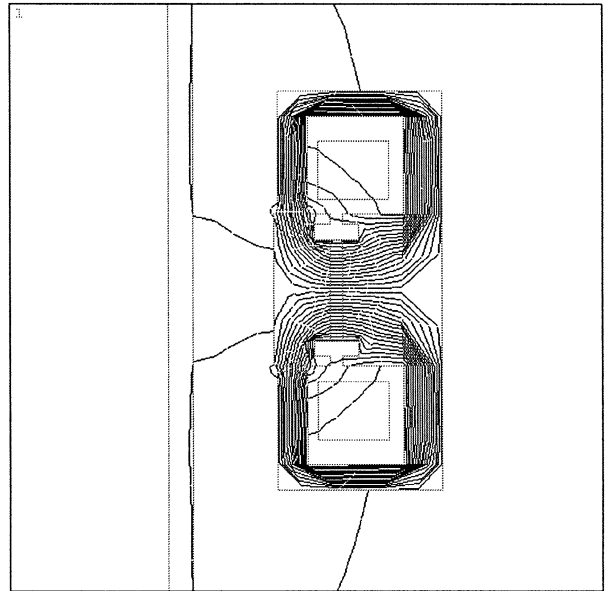

FIGURE 9

2-D flux lines. analysis of the magnetic behavior of the device was carried out by using a finite elements code (ANSYS ${ }^{\circledR}$ ) relating the value of the electric current running in the coils and the value of the magnetic field in the fluid. An axial symmetric model was employed since the objective of the analysis was only to optimize the geometry of the magnetic circuit. Figures 8 and 9 show the values of the magnetic field and the flux lines in the device for a current density $J_{S}=7 \mathrm{~A} / \mathrm{mm}^{2}$.

All material magnetic properties were assumed linear, i.e., constant relative magnetic permeability, $\mu_{r}=2000$ for steel and $\mu_{r}=5$ for the MR fluid. The linear relationship between the current density in the coils (measured in $\mathrm{A} / \mathrm{mm}^{2}$ ) and the magnetic field in the MR fluid $B_{\mathrm{MR}}$ (measured in Tesla) is expressed by the following equation:

$$
B_{\mathrm{MR}}=7.15 \cdot 10^{-2} J_{S}
$$

The bearing magnetic permeability was neglected because of the discontinuous structure (i.e., balls and plastic cage).

\section{THE TEST RIG}

The rotor, which was supplied with the Bently Nevada Rotor Kit (see Figure 10), is a slender $10 \mathrm{~mm}$ diameter shaft, $560 \mathrm{~mm}$ long. One or two discs, of $0.8 \mathrm{~kg}$ each, can be fixed on the shaft. These discs have threaded holes at a radius of $30 \mathrm{~mm}$ in order to create the desired unbalance inserting appropriate screwweights. The shaft, supported by two adjustable oilite bearings, can reach a maximum rotational speed of $10.000 \mathrm{rpm}$. The RotorKit bench is equipped with eddy current probes for relative displacement measurements (3300 NSV ${ }^{\mathrm{TM}}$ Probe Proximitor ${ }^{\circledR}$ ).

The damper is supported by an aluminium ring connected to the pedestal of the Rotor-Kit, and is mounted on the shaft by means of the aluminium bush shown in Figure 6. It is connected

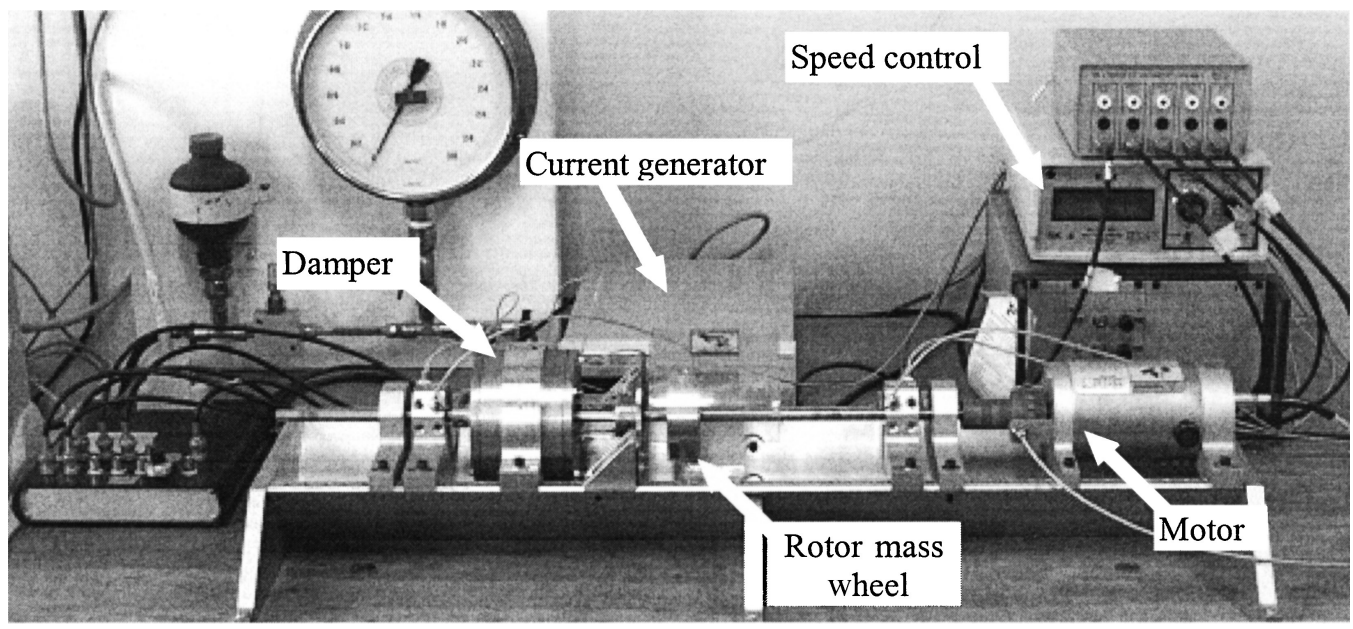

FIGURE 10

The test-rig. 
to a current generator (Hewlett-Packard E3611A DC power supply) in order to feed the coil with a maximum constant current of $1.5 \mathrm{~A}$.

\section{PRELIMINARY TESTS}

Preliminary tests have been performed. In the tested configuration, the oilite bearings were $440 \mathrm{~mm}$ apart and the damper was placed at a distance of $110 \mathrm{~mm}$ from one of them. The disk was then unbalanced with a $7 \mathrm{~g}$ grain. This value appears quite high in relation to the shaft and disk dimensions. During the numerical simulations it was observed that such unbalance is necessary to test the characteristics of the MR fluid used since a lighter grain would not be able to break the columnar structure created in the fluid and activate the damper unless the yield stress was extremely smaller.

The sensors were placed at a distance of $3 \mathrm{~cm}$ from the oilite bearing farther from the damper. They were not placed too close to the whirling disk in order to avoid off-scale problems since the tests are characterized by great deformation of the shaft.

Rotational speed varying from 1000-5000 rpm were investigated at first, verifying the behavior of the damper with no field applied and then feeding the coil with two different values of current: $0.5 \mathrm{~A}$ and $1 \mathrm{~A}$. The calculated corresponding fields generated in the fluid, according to Equation (8), are $0.25 \mathrm{~T}$ and $0.12 \mathrm{~T}$, respectively.

The corresponding yield stress values of $8000 \mathrm{~Pa}$ and $3000 \mathrm{~Pa}$ are found from the relation shown in Figure 11. Figure 12 shows the value of the radial displacement of the shaft, at section 1 , versus the rotational speed. The magnetic field takes effect only when it becomes greater than $0.12 \mathrm{~T}$ and a significant reduction of the radial displacement of the shaft takes place for a yield stress equal to $8000 \mathrm{~Pa}$. Compared to the numerical simulation, the behavior is similar to the expected one but there is a difference in the value of the effective yield stress; that can have different explanations. One explanation is related to the problem encountered in completely filling the gap as there might be some vacuum zones increasing the value of the current needed to generate the field and the corresponding yield stress. Such problems

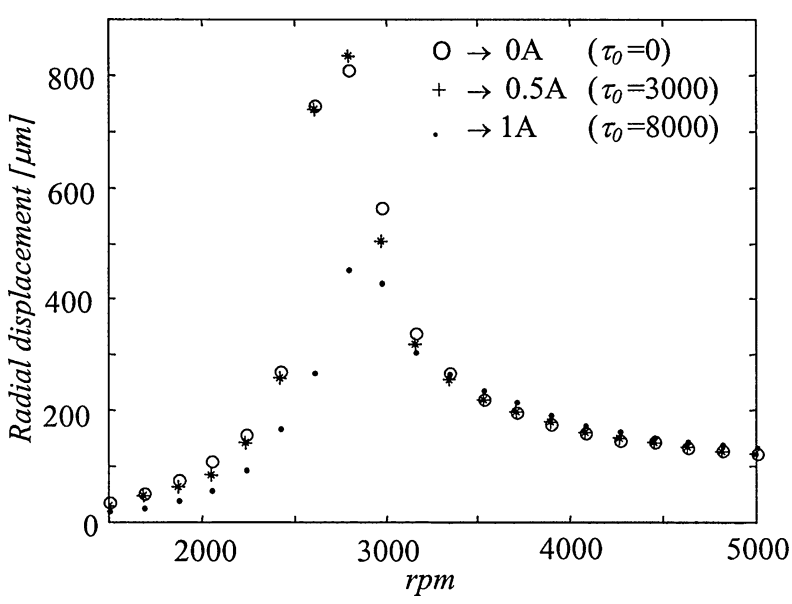

FIGURE 12

First experimental results.

will be overcome in the next experimental campaign. Moreover, since during the motion the magnetic field in the device is not axial symmetric, the magnetic field in the fluid and, consequently the yield stress, might vary significantly along the angular coordinate. Finally, there might be some nonlinearities not taken into account in the analytical model.

\section{CONCLUSIONS}

In this article, a MR fluid squeeze film damper has been presented. The MR damper takes advantage of the synergic effects of a variable viscosity squeeze film damper and a magnetic damper. The simulation of the operating conditions, limited to the fluid squeeze effect, has given indication for the damper design. The preliminary experimental results obtained with the developed device show its effectiveness in dampening the rotor vibrations, controlling its dynamic characteristics by simply varying the current in the magnetic coils. An extensive experimental investigation is planned for the near future on the described device and on a modified configuration.

\section{NOMENCLATURE}

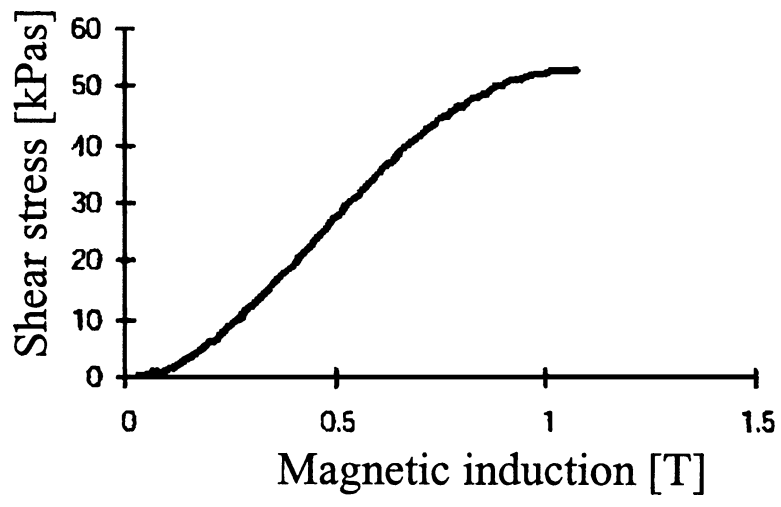

FIGURE 11

Shear stress vs. magnetic induction in the fluid.

$\begin{array}{ll}a & \text { distance damper-shaft end } \\ B_{M R} & \text { magnetic field in the MR fluid } \\ c & \text { bearing radial clearance } \\ e & \text { bearing eccentricity } \\ h & \text { film thickness } \\ J_{S} & \text { current density in the coils } \\ k_{11}, k_{12}, k_{22} & \text { shaft stiffness coefficients } \\ k_{s c} & \text { squirrel cage stiffness coefficient } \\ l & \text { shaft length } \\ L & \text { bearing axial length } \\ m_{d} & \text { disk mass } \\ m_{g} & \text { grain mass } \\ M_{1} & \text { unbalanced disk mass }\left(m_{d}+m_{g}\right)\end{array}$




$\begin{array}{ll}M_{2} & \text { journal mass } \\ p, p^{*} & \text { pressure } \\ q & \text { volume flow rate/axial length } \\ R, R_{o}, R & \text { journal radius, inner and outer radii } \\ u, u^{*}, v, w & \text { fluid velocity in } x, y, z \text { directions } \\ x & \text { spatial coordinate, sliding direction } \\ y, y^{*} & \text { spatial coordinate, direction across film } \\ X_{1}, Y_{1} & \text { coordinate system (disk location) } \\ X_{2}, Y_{2} & \text { coordinate system (damper location) } \\ z & \text { spatial coordinate, normal to } x, y \\ \beta & \text { mass centre rotation with respect to } X_{1} \\ \delta & \text { unbalance distance of mass centre } \\ \varepsilon & \text { eccentricity ratio }(e / c) \\ \theta & \text { angular coordinate }(x / R) \\ \mu & \text { fluid viscosity } \\ \mu_{0} & \text { vacuum magnetic permeability } \\ \mu_{r} & \text { relative magnetic permeability } \\ \tau_{0}, \tau_{0}^{*} & \text { yield shear stress } \\ \omega & \text { precession speed } \\ ()^{*} & \text { dimensionless quantity }\end{array}$

\section{REFERENCES}

Bolter, R., and Janocha, H. 1997. Design rules for MR fluid actuators in different working modes. SPIE 3045:148-159.

Bonneau, O., and Frene, J. 1997. Non-linear behavior of a flexible shaft partly supported by a squeeze film damper. Wear 206:244250.

Carlson, J. D., and Weiss, K. D. 1994. A growing attraction to magnetic fluids. Machine Design 66(15):61-64.

Carmignani, C., and Forte, P. 2001. Active squeeze film dampers in rotordynamics. International Journal of Applied Mechanics and Engineering 6(4):1061-1072.

Dimarogonas, A. D., and Kollias, A. 1992. Electrorheological fluidcontrolled "smart" journal bearings. STLE Tribology Transactions 35(4):611-618.
Jolly, M. R., Bender, J. W., and Carlson, J. D. 1998. Properties and applications of commercial magnetorheological fluids. Spie 5th Annual International Symposium on Smart Structures and Materials, March 15, San Diego, CA.

Jolly, M. R., and Carlson, J. D. 1996. Controllable squeeze film damping using magnetorheological fluid. Proceedings of the 5th International Conference on New Actuators, June 26-28, Bremen, Germany, 333 336.

Jung, S.Y., and Choi, S.B. 1995. Analysis of a short squeeze-film damper operating with electrorheological fluids. STLE Tribology Transactions 38(4):857-862.

Kordonsky, W. I. 1993. Magnetorheological effect as a base of new devices and technologies. Journal of Magnetism and Magnetic Materials 122:395-398.

Morishita, S., and An, Y. K. 1996. On dynamic characteristics of ER fluid squeeze film damper. JSME International Journal, series C, 39(4):702-707.

Morishita, S., and Tamaki, U. 1993. ER fluid applications to vibration control devices and an adaptive neural-net controller. Journal of Intelligent Material Systems and Structures 4:366-372.

Nikolakopoulos, P. G., and Papadopoulos, C.A. 1998. Controllable high speed journal bearings, lubricated with electro-rheological fluids. An analytical and experimental approach. Tribology International 31(5):225-234.

Shakeri, C., Noori, M. N., and Hou, Z. 1996. Smart materials and structures, a review. Proceedings of the 1996 4th Materials Engineering Conference, part 2, Washington, D.C. Materials for the New Millennium Proceedings of the Materials Engineering Conference 2, ASCE, New York, 863-876.

Tichy, J. A. 1991. Hydrodynamic lubrication theory for the Bingham plastic flow model. Journal of Rheology, 35(4):477-496.

Tichy, J. A. 1993. Behavior of a squeeze film damper with electrorheological fluid. STLE Tribology Transactions 36(1):127-133.

Weiss, P. K., Carlson, J. D., Duclos, G. T., Chrzan, M. J., and Margida, A. J. 1993. High strength magneto- and electrorheological fluids. SAE paper $932451,1993$.

Zhu, C., Robb, D. A., and Ewins, D. J. 2001. Magnetorheological fluid dampers for rotor vibration control. AIAA 1469:2121-2127. 

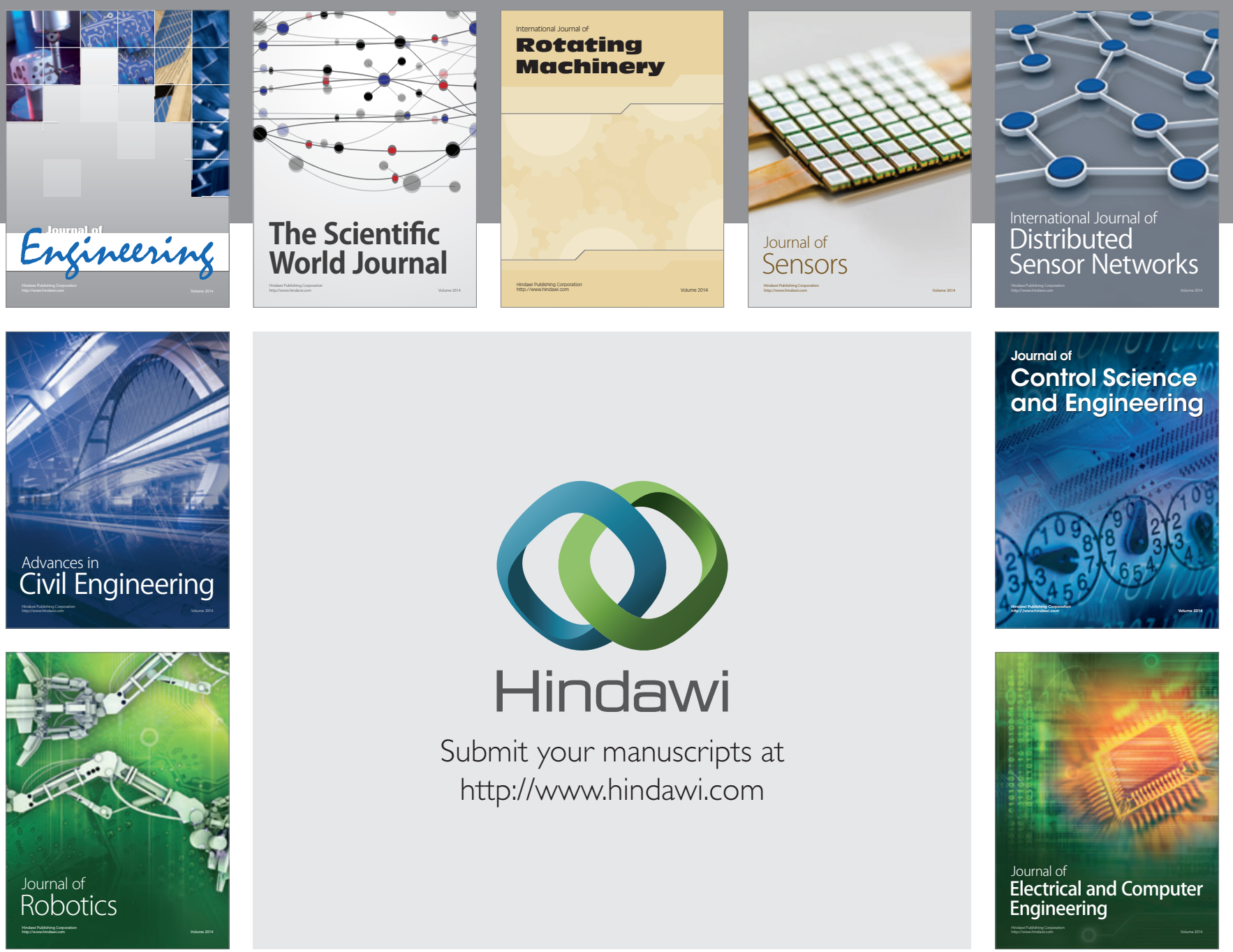

Submit your manuscripts at

http://www.hindawi.com
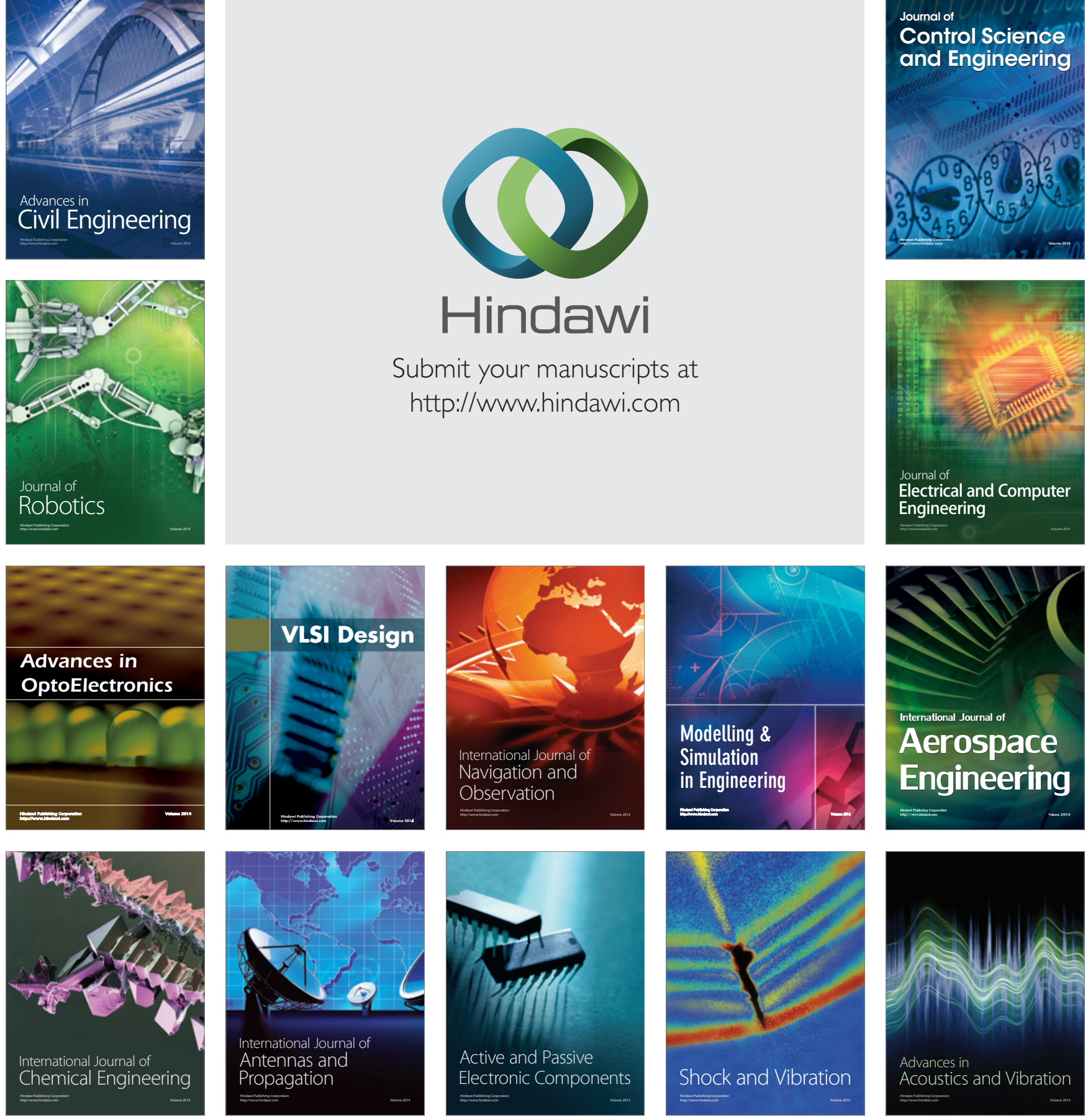